\title{
Helices at Interfaces
}

\author{
Gi-Moon Nam ${ }^{1,2}$, Nam-Kyung Lee ${ }^{1,2}$, Hervé Mohrbach ${ }^{1,3}$, Albert Johner ${ }^{1}$ and Igor M. Kulić ${ }^{1}$ \\ 1 Institut Charles Sadron, CNRS 23 rue du Loess 67034 Strasbourg cedex 2, France \\ 2 Institute of Fundamental Physics, Department of Physics, Sejong University, Seoul 143-743, Korea. \\ ' 3 Groupe BioPhysStat, Université de Lorraine, 57078 Metz, France
}

PACS $82.35 . \mathrm{Pq}-$ Biopolymers.

PACS 87.16.Ka - Filaments, microtubules, their networks, and supramolecular assemblies.

\begin{abstract}
Helically coiled filaments are a frequent motif in nature. In situations commonly encountered in experiments coiled helices are squeezed flat onto two dimensional surfaces. Under such 2-D confinement helices form "squeelices" - peculiar squeezed conformations often resembling looped waves, spirals or circles. Using theory and Monte-Carlo simulations we illuminate here the mechanics and the unusual statistical mechanics of confined helices and show that their fluctuations can be understood in terms of moving and interacting discrete particle-like entities - the "twist-kinks". We show that confined filaments can thermally switch between discrete topological twist quantized states, with some of the states exhibiting dramatically enhanced circularization probability while others displaying surprising hyperflexibility.
\end{abstract}

Introduction. - Helically coiled filaments are found ' everywhere in living nature. The list of examples is close to innumerable with the most prominent ones: FtsZ [1], Mrb 2, bacterial flagella [3, 4, tropomyosin [5] and intermediate filaments [6. More recently microtubules were suggested to spontaneously form large scale superhelices [7,8]. Even whole microorganisms exhibit helicity inherited from their constituent filaments [9]. The superhelicity of filaments is in some cases of strong evo' lutionary benefit as in the example of swimming bacte'ria utilizing the rotational motion of their helical flagel' lar filament for propulsion 10 and tropomyosin's helical ' "Gestalt-binding" around actin [5]. In other cases, like . for microtubules the purpose of superhelicity remains so far unknown 8]. Paralleling biological evolution artificial, man made helically coiled structures have been created including coiled carbon nanotubes [11, DNA nanotubes 12], coiled helical organic micelles [13].

As happens often in experiments our justified desire to simplify observation conditions by confining filaments to a surface (coinciding with the focal plane) changes the physical properties of the underlying objects in initially unanticipated but physically rather interesting manner. With filament helices being such a profoundly ubiquitous structure it is the purpose of this work to investigate the rich physical effects of helical filaments confinement. As we will show, the confinement changes dramatically the shape as well as statistical mechanics of the confined he- lix generating several notable and surprising effects: a) Enhancement of cyclisation probability, b) Enhancement of end-to-end fluctuations and c) Generation of conformational multistability (despite apparent linearity of constitutive relations). We will see that the conformational dynamics of confined helices is most naturally described in terms of discrete particle like entities - the "twist kinks", cf. Fig. 1. We show that these "twist kinks" are completely analogous to overdamped Sine-Gordon-kinks from soliton physics [14 as well as loops in stretched elastic filaments [15. These analogies will help us to rather intuitively develop a phenomenological understanding of the underlying physics.

The Phenomenology of Squeezed Helices. Confined biofilaments throughout literature exhibit often abnormal, wavy, spiral, and circular shapes that appear not be rationalized by the conventional Worm Like Chain model. This riddle of peculiar filament shapes is the starting point of our investigation. In this letter, we propose a new augmented model of confined intrinsically curved and twisted chains that leads to a variety of 2-D shapes matching experimental observations. The corresponding 2-D geometrical curves we call squeezed helices or more briefly - squeelices. Filaments are modelled as 2-D confined Helical Worm Like Chains (cHWLC) with bending modulus $B$, twist modulus $C$ and a preferred curvature $\omega_{1}$ and twist $\omega_{3}$. 
Phenomenologically (as detailed further below) the main effect of confinement is to introduce narrow regions where the twist is highly concentrated and the curvature flips, cf. Fig. 1. These curvature flip points we will call "twist-kinks" in reference to the concept of kinks in soliton physics [14. Depending on the control parameter $\gamma \sim \frac{B \omega_{1}^{2}}{C \omega_{3}^{2}}$ two regimes can be distinguished:

i) $\gamma>1$ : Twist kinks having a positive self-energy are essentially expelled and can only be thermally activated.

ii) $\gamma<1$ : Twist kinks have a negative self-energy and the ground state involves a finite density of twist kinks.

In both cases the generic shape motive is a circular arc section or in special cases closed circles. If present, twist kinks separate arcs of opposite curvature orientations resulting in a wavy undulatory 2-D shape. For a squeelix with $\gamma>1$, twist expulsion results in a circular arc shaped ground state -a feature that can favor the occurrence of closed filaments, which are very unlikely in the free helical state (in absence of confinement).

Our study is based on the analytical analysis of the confined helical WLC Hamiltonian and numerical Monte Carlo simulations using the density of states method [16]. The simulations, primarily designed to illustrate circularization enhancement, focus on the most curious case of twist expulsion where the ground state becomes circular up to (weak and localized) edge effects. In this regime, besides the ground state, the chain will also comprise excited (wavy looking) states involving a discrete number of thermally activated twist-kinks. Because an isolated twistkink almost freely diffuses along the filament an excited state can be "hyperflexible" meaning that large shape fluctuations are induced by simple translational sliding motion of the twist-kink along the contour. For sets of parameters not resulting in twist expulsion $(\gamma<1)$, the ground state itself is periodically wavy. This case where a squeezed helix forms a wavy periodic 2-D structure appears less exciting as effects like circularization and hyper-flexibility will be absent. For this reason we will entirely focus in this very first study on the more illuminating and surprising limit $\gamma>1$.

A note for the experimentalist concerning non-ideal filaments (in real world experiments) seems appropriate here. Bacterial flagella and microtubules are known to be helical filaments, but their mechanical characteristics are generally (in experimental practice) not quite uniform along the filament. There can be frozen-in lattice defects like a jump in the number of protofilaments for microtubules [17] or annealed defects like a boundary between different polymorphic states in bacterial flagella. In the cases of interest the mechanical parameters are uniform within some correlation length, which may even be larger for cleanly prepared filaments than the contour length under scrutiny. Our model is generic in the sense that it applies to a uniform sub-filament. A clean observation and comparison of the shapes described below further supposes that shape equilibration is possible with uniform mechanical param- eters.
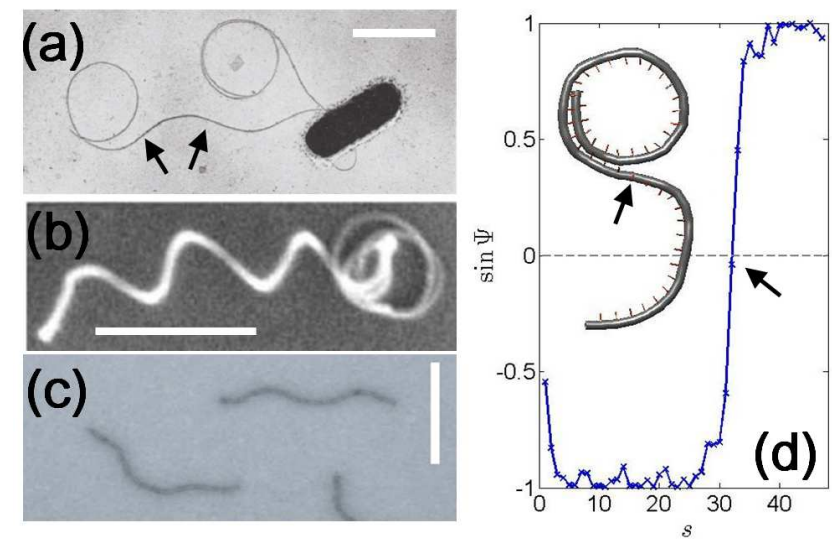

Fig. 1: Squeezed helical filaments ("squeelices") are frequently observed under experimental conditions. a) Polymorphic helical bacterial flagella emerging from a bacterium [18] (Scale bar $1.3 \mu \mathrm{m})$. b) A coiled carbon nanotube when adsorbed onto the substrate from [11, (Scale bar $0.5 \mu \mathrm{m})$ c) Helical DNA nanotubes 12 (Scale bar, $0.5 \mu \mathrm{m})$. c) In simulations squeelices appear in coexisting wavy-circular shapes and display discrete "twist kinks" (curvature inversion points) indicated by arrows. Twist kinks - discrete non-linear elementary excitations of confined helices - are characterized by a rapid inversion of the normal vector along the contour and by a localized twist-angle $\psi$ variation

The Squeezed Helical Chain Model: Free vs Confined. - The variety of squeelical shapes in Figs. 1 and 4 can be understood by simple considerations about their energy. The elastic energy of a helical wormlike chain can be written as a function of the local curvatures $\Omega_{1,2}$ and twist $\Omega_{3}$ as:

$$
E=\frac{1}{2} \int_{-L / 2}^{L / 2} B\left[\left(\Omega_{1}-\omega_{1}\right)^{2}+\Omega_{2}^{2}\right]+C\left(\Omega_{3}-\omega_{3}\right)^{2} d s,
$$

The 3 -D ground state is a helix of radius $R=\frac{\omega_{1}}{\omega_{1}^{2}+\omega_{3}^{2}}$ and pitch $H=\frac{2 \pi \omega_{3}}{\omega_{1}^{2}+\omega_{3}^{2}}$ satisfying the preferred curvature and twist everywhere. To proceed and access the filament shapes, it is convenient to express the $\Omega_{i}$ through the Euler angles $\Omega_{1}=\phi^{\prime} \sin \theta \sin \psi+\theta^{\prime} \cos \psi, \Omega_{2}=$ $\phi^{\prime} \sin \theta \cos \psi-\theta^{\prime} \sin \psi$ and $\Omega_{3}=\phi^{\prime} \cos \theta+\psi^{\prime}$. We constrain the chain to a plane by imposing $\theta=\pi / 2$. The elastic energy of such a confined squeelix can be recast as:

$$
E=\frac{B}{2} \int_{-L / 2}^{L / 2}\left(\left(\phi^{\prime}\right)^{2}-2 \omega_{1} \phi^{\prime} \sin \psi+\omega_{1}^{2}+c\left(\psi^{\prime}-\omega_{3}\right)^{2}\right) d s
$$

where $c=C / B$ and $s$ is the arc length parameter along the chain. Now $\phi^{2}=\Omega_{1}^{2}+\Omega_{2}^{2} \equiv \kappa^{2}$ gives the local curvature of the chain in the plane and $\psi^{\prime}=\Omega_{3}$ is its local twist. Interestingly it is seen from $\mathrm{Eq} 2$ that the curvature and 
the twist are now coupled through $\omega_{1}$ - which turns out to lie at the heart of most phenomena discussed here. The optimal shape satisfies the Euler-Lagrange equations:

$$
\begin{gathered}
\phi^{\prime}=\omega_{1} \sin \psi . \\
\psi^{\prime \prime}+\frac{\omega_{1}^{2}}{2 c} \sin 2 \psi=0 .
\end{gathered}
$$

If no external torque is applied on the chain, the twist must satisfy the boundary conditions $\psi^{\prime}(-L / 2)=\psi^{\prime}(L / 2)=$ $\omega_{3}$. A quick glance at Eq. 4 reveals that $\psi(s)$ is the solution of a pendulum equation and thus is a Jacobi elliptic function [19]. Eq. 3 shows that under confinement the curvature becomes "slaved" by the twist and is no more an independent parameter as for a free chain. This obviously is at the origin of the localization of twist. For the sake of simplicity, instead of solving Eqs. 344 directly, we will gain more physical insight by rewriting Eq. 2] in terms of a WLC under tension (WLC-T) for which it is easy to develop intuition. For this we plug in Eq 3 into the energy Eq2 and introduce the new angle $\vartheta=2 \psi-\pi$ to get:

$$
\begin{aligned}
E(\vartheta) & =\int_{-L / 2}^{L / 2}\left(\frac{1}{2} \tilde{A} \vartheta^{2}+\tilde{F}(1-\cos \vartheta)\right) d s \\
& -\frac{M}{2}[\vartheta(L / 2)-\vartheta(-L / 2)],
\end{aligned}
$$

up to an inessential constant term. Here we introduced $\tilde{A}=C / 4$ as the effective bending modulus of the WLC-T and $\tilde{F}=B \omega_{1}^{2} / 4$ as the effective external force acting on it. The integral term is precisely the WLC-T energy. The last term with $M=\omega_{3} C$ represents the torque exerted on both ends of the chain. If large enough, this term enforces $n$ extra trapped turns/loops $\vartheta(L / 2)-\vartheta(-L / 2)=2 \pi n$ or in the $\psi$ representation, $n$ twist-kinks along the squeelix.

The phenomenology of the stretched chain is common knowledge [15, which makes the mapping attractive. The decay length of any localized distortion (correlation length) $\lambda$ is the loop size of a loop grown against the tension $F$ (in the loop picture) or kinks-spatial extension (in the twist-kink picture):

$$
\lambda=\sqrt{\tilde{A} / \tilde{F}}=\frac{1}{\omega_{1}} \sqrt{\frac{C}{B}}
$$

The WLC-T loop stores a typical energy $\sim \lambda \tilde{F}$. If the work of the external torques $2 \pi M$ per loop reduces sufficiently the loop energy, loops form spontaneously (otherwise they can be thermally activated). We hence conclude that the ground state is wavy for $M \gtrsim \lambda \tilde{F}$, which translates into $1 \gtrsim \frac{B \omega_{1}^{2}}{C \omega_{3}^{2}}$ in the cHWLC language, and circular otherwise.

An overestimate of the energy stored in the loop $2 \sqrt{2} \pi \sqrt{\tilde{A} \tilde{F}}$ is obtained assuming a circular loop. The WLC-T featuring loops in the long chain limit has been studied in detail in ref. [15] from which we borrow the more precise expression $E_{\text {loop }}=8 \sqrt{\tilde{A} \tilde{F}}$. After subtracting the work of the torques we arrive at the effective self-energy of a single twist-kink:

$$
E_{1 k i n k}=(\sqrt{\gamma}-1) \pi C \omega_{3}
$$

where

$$
\gamma=\frac{4 B \omega_{1}^{2}}{\pi^{2} C \omega_{3}^{2}}
$$

is the twist-kink expulsion parameter introduced previously. The shape of an isolated twist-kink is easily obtained upon integrating Eq, for a twist kink localized around $s=0$ :

$$
\sin \psi(s)=\tanh \frac{s}{\lambda} \quad \text { and } \quad \phi^{\prime}(s)=\omega_{1} \tanh \frac{s}{\lambda}
$$

a result we could also have transposed from [15]. From Eq9 it is clear that provided $L \gg \lambda$ the twist kink is localized and separates two curvature flipped regions of almost constant curvature $\approx \omega_{1}[20$.

Let us now turn to the main focus of this paper, the case of twist expulsion $(\gamma>1)$, which maps to the ground state to a WLC-T without loops. In a consistent thermodynamic picture the twist-kink density should be calculated including shape fluctuations, in particular the coupling of the twist kink to small thermal deformations. These linear fluctuations -which in the soliton picture are often referred to as "phonons" 14 - are expected to only moderately renormalize the kinks free energy (by $\sim k_{B} T$ per kink). Fortunately intuitive reasoning and simple geometry allows us to calculate filament's shape fluctuations associated with the free sliding motion of the narrow twist-kink along the filament in the excited state. The associated soft mode (kink's position) is at the origin of the surprisingly large fluctuations in the excited state reported in simulations below.

The opposite regime $\gamma<1$ where the squeelix shapes become wavy can be described along similar lines with the twist kink density being limited by mutual kinkkink repulsion. Generally speaking for $\gamma<1$ the gas of twist kinks seizes to be ideal, giving rise to reduced compressibility and in turn weaker extension fluctuations of the squeelix. It can be shown that the pair repulsion between twist-kinks decreases with their distance $d$ as $U_{\text {int }} \sim \pi C \omega_{3} \sqrt{\gamma} f(d / \lambda)$ with $\mathrm{f}(x) \sim 1 / x$ for $x \ll 1$ and $f(x) \sim e^{-x / 2}$ for $x \gg 1$. The dense twist kink regime deserves special consideration, a more detailed description explicitly involving Jacobi functions will be given elsewhere.

Simulation. - Equipped with this intuitive picture of a squeezed chain as a collection of weakly interacting 1D "particles" (twist-kinks) whose positions along the contour relate to chain's deformations in simple manner (Eq. 9] we move on to investigate the formulated hypotheses with a Wang-Landau type Monte-Carlo simulation [16. We model the system as a discrete helical WLC consisting of 
$N$-monomers 21] subjected to a discrete version of the Hamiltonian given in Eq, The chain is further confined by a harmonic potential so that each monomer located at distance $z$ away from the $z=0$ surface experiences potential $E_{\text {conf }}=K z^{2}$ with $K=25 k_{B} T / b^{2}$, with the bond length set as $b=1$. For $K=0$ the confinement vanishes and we recover the free chain statistics in 3-D.

The main output of the simulation is the density of states (DOS) [16. The equilibrium conformational statistics can be obtained from the DOS after Boltzmann weighting according to the Hamiltonian. In addition to the energy $E$, the joint DOS $g(E, D, n)$ was sampled with respect to the end-to-end distance $D$ and to the "number of kinks" $n$, where $n=\frac{1}{\pi} \sum_{i}^{N-2} \Omega_{3, i}$. The latter is indeed a statistical analogue of the discrete twist kink number in the theoretical consideration above. Sampling of twodimensional or higher histograms is often computationally more demanding than one-dimensional histograms. For efficient sampling, we used the global update method introduced by 27. The simulations were performed for several chain lengths, $N=16,32,64$ and with fixed mechanical parameters $B=50, C=25 b k_{B} T$ throughout. The essential information on the confined cHWLC gained from our simulations are summarized in free energy maps $F(n, D)$ (see fig凹(b)).

Results. - Figure 2 demonstrates the typical changes of chain's conformation triggered by confinement. For values $\gamma>1$, the confined helix assumes an approximately circular shape as shown in Fig,2(a). The binormal vectors pointing all in the same direction (in red) indicate that twist is expelled from the chain. The normal vectors are in-plane and pointing to the center of circle (in blue). For $\gamma<1$, on the other hand, the chain assumes a stretched and twisted undulatory wavy shape as seen in Fig 2 (b). The wavy shape consists of a discrete number of localized twist kinks as anticipated from theory. At the location of twist-kinks the in-plane normal vectors and the in plane curvature $\phi^{\prime}$ flip their signs. The curvature between the twist kinks is approximately constant $\approx \omega_{1}$ as expected from the theoretical shape of single twist kinks Eq. 9

Hypercyclisation. The phenomenological theoretical arguments above predict that a significant circularization enhancement should be observable for a twist-expelling chain $(\gamma>1)$. We subject this hypothesis to a simulation test. For the geometric parameters $\omega_{1} N=4.8$ and $\omega_{3} N=3.2$ and elastic constant ratio $B / C=2$ the twist expulsion parameter is well over unity $\gamma=1.82$. In absence of confinement, a chain with these parameters assumes a helical shape with a radius given by $R=0.14 N b$ and a pitch $H=0.62 N b$. When on the other hand such a chain becomes strongly confined to $2 \mathrm{D}$, it morphs into a slightly open but approximately circular shape with an end-to-end distance approximately given by $\frac{D_{0}}{N b} \approx\left|\frac{2}{\omega_{1} N} \sin \left(\frac{\omega_{1} N}{2}\right)\right|=0.28$. In such a case, the newly formed ground state under confinement seems to



Fig. 2: Formation of two different classes of conformations of a confined helix depending on the twist expulsion parameter $\gamma$. The contour is represented by a tube with a normal (in red) and a binormal vector (in blue). (a) and (b) typical helical chains in 3-D before confinement. When confined, (a') untwisted circularized shapes $(\gamma>1)$ and $\left(b^{\prime}\right)$ twisted and wavy shapes $(\gamma<1)$ emerge.

promote circularization. To quantify the circularization enhancement we define the circularization probability $P_{d}^{\bigcirc}\left(D_{c a p}\right)$ given by the likelihood to find both chain ends within a fixed (small) capture distance $D_{c a p}$

$$
P_{d}^{\bigcirc}\left(D_{c a p}\right)=\int_{0}^{D_{c a p}} p_{d}(D) d D .
$$

where $p_{d}(D)=\frac{1}{Z} \iint g(E, D, n) e^{-E / k_{B} T} d E d n, Z=$ $\iiint g(E, D, n) e^{-E / k_{B} T} d E d n d D$ is the radial probability density for a given end-distance $D$ and the index $d=2$ or 3 stands for the dimensionality of the chains embedding (confined in 2D or free in $3 \mathrm{D}$ ). Fig 3 shows the radial distribution functions $p_{2}(D)(2-\mathrm{D})$ and $p_{3}(D)(3-\mathrm{D})$ at various temperatures. The maximum of $p_{3}(D)$ (blue line) is located at about $D=0.55 \mathrm{Nb}$ close to the expected helical pitch. In contrast the largest population of the confined chains is located around the peak of $p_{2}(D)$ (blue line) at $D_{0} / N b \approx 0.28$ which corresponds to the expected 2-d circular ground state. At higher temperature, chain becomes more flexible and the end-to-end distribution broadens due to the thermal fluctuations.

Integrating the probability density Eq 10 we can estimate the circularisation enhancement factor $f^{\bigcirc}=$ $P_{2}^{\bigcirc}\left(D_{\text {cap }}\right) / P_{3}^{\bigcirc}\left(D_{\text {cap }}\right)$ when the chain is squeezed to $2 \mathrm{D}$ as a function of the chains bending persistence length $l_{p}=B / k_{B} T$. As suggested on theoretical grounds the closing probability is greatly enhanced due to the confinement at lower temperatures (or increased chain stiffness) - with $f \bigcirc$ well in excess of 1000 (Fig[3 ).

Multistability and Hyperflexibilty. The theoretical considerations of multi-kink solutions presented above indicate that confining a helical chain should generate a complex energy landscape with many coexisting discrete 

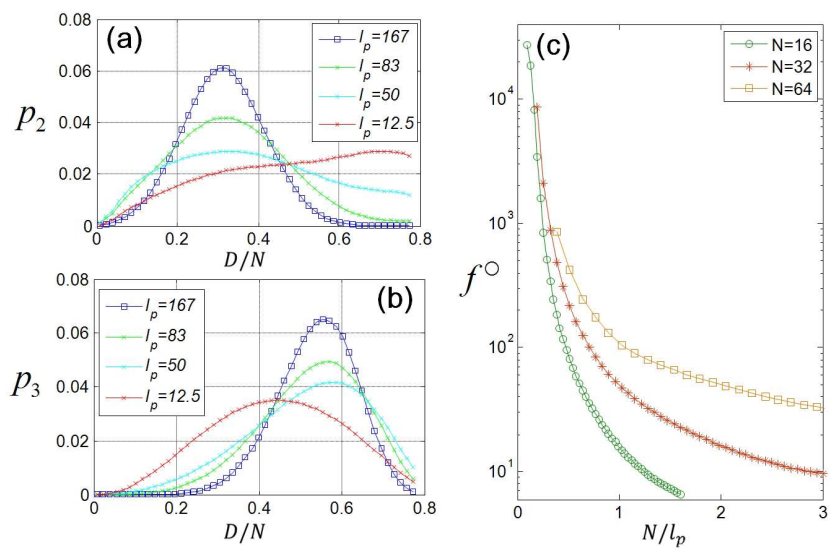

Fig. 3: Confinement enhances circularization. The end-toend distance $D$ distribution as a function of chain's bending persistence length $l_{p}=B / k_{B} T$ (a) in $2 \mathrm{D}$ (a) and (b) 3D $\left(N=32 b, \omega_{1}=0.15 b^{-1}, \omega_{3}=0.1 b^{-1}\right.$, and $\left.\gamma=1.82>1\right)$. The enhancement of circularization probability upon confinement $f^{\bigcirc}=P_{2 d}^{\bigcirc}\left(D_{\text {cap }}\right) / P_{3 d}^{\bigcirc}\left(D_{\text {cap }}\right)$ as a function of the normalized chain length $N b / l_{p}$ with capture distance $D_{\text {cap }}=0.5 b$.

states. Some of these states, in particular those comprising only a few kinks, should exhibit anomalous hyperflexible behavior due to the energetically cheap displacement of kinks. For instance the motion of a single kink on a chain of length $L$ and curvature $\omega_{1}$ satisfying a close to "resonance" condition $\omega_{1} \approx k \frac{2 \pi}{L}(k=1,2 \ldots)$ should give rise to most dramatic end fluctuation effects.

To test this we calculate the joint DOS of three variables $g(E, D, n)$ and the free energy landscape given by $\beta F(D, n)=-\ln \left[\sum_{E} g(E, D, n) e^{-\beta E}\right]$ (Fig. 4a,b). For parameters $\omega_{1}=0.26 b^{-1}$ and $\omega_{3}=0.1 b^{-1}$, in 3D (confinement free case) we would obtain a helix with helical radius $R=8.09 b$ and pitch $H=3.35 b$. In contrast to that as expected from the large twist expulsion parameter $\gamma=5.47$ the chain's 2D ground state shape under confinement becomes a coiled double circle with roughly two turns. Besides the ground state, the free-energy as a function of $n$ indicates the existence of further ("excited") low energy states with $n=1,2, \ldots$. (Fig. 4). These states with distinct $n$ are separated by small free energy barriers. The first excited state $n=1$ for instance has a free energy difference of only $\Delta G_{1 k i n k} \approx 4 k_{B} T$ a figure that is not far from the theoretical estimate of the kink free energy $\Delta G_{1 k i n k}=E_{1 k i n k}-k_{B} T \ln N \approx 6.6 k_{B} T$ where the $\ln N$ $(\mathrm{N}=48)$ term accounts for kinks positional entropy gain along the discrete positions of the chain [22].

The $n=1$ state (cf. Fig. 4(c)) exhibits an enhanced end-end distance fluctuation $\left\langle(\delta D)^{2}\right\rangle$ in phenomenological agreement with the mobile kink interpretation. For the given length $(L=48 b)$ and curvature we expect an almost flat free energy landscape as a function function the end-end distance $D$ in the range $D \in\left[0, D_{\max }\right]$. Here $D_{\max } \approx 4 \omega_{1}^{-1}=15.4 b$ is the maximal extension for the $n=1$ state which is reached when the twist-kink is lo-
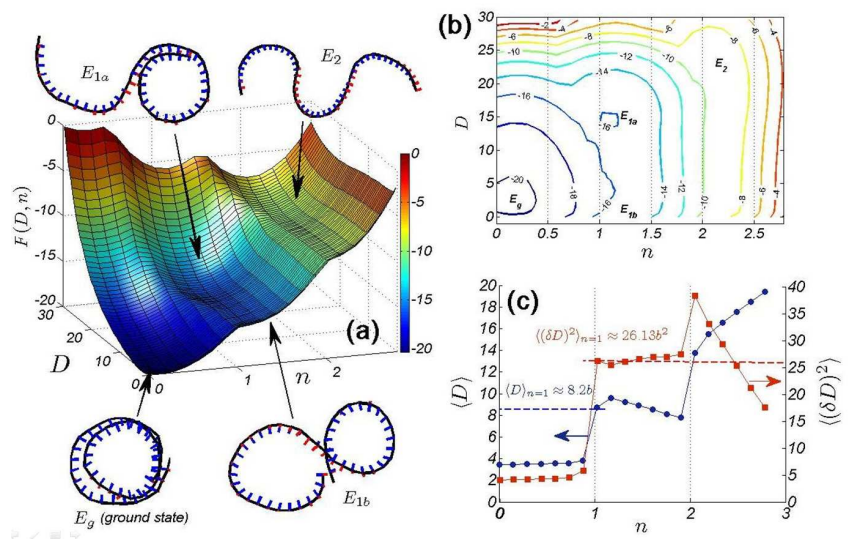

Fig. 4: (a)The free energy landscape of a fluctuating squeelix as a function of the end-to-end distance $D$ and the number of twist kinks $n\left(N=48 b, \omega_{1}=0.26 b^{-1}, \omega_{3}=0.1 b^{-1}\right.$, and $\gamma=5.47>1)$. The ground state $E_{g}(n=0)$ is a two-turn circular shape without kinks. The excited states $n \geq 1$ comprise one or more very mobile kinks giving rose to strong shape fluctuations with states $E_{1 a}$ and $E_{1 b}$ having identical energies. (d) Contour plot of free energy for 2 -d chain with indicated values free energy values in units of $k_{B} T$. (c) Mean of end-to-end distance $\langle D\rangle$ and its standard deviation $\left\langle(\delta D)^{2}\right\rangle$ as a function of $n$ (dual axis graph).

cated at a position $\approx L / 4$ from any of the borders- as seen from straight forward geometric reasoning. The standard deviation expected in this case $\left\langle(\delta D)^{2}\right\rangle_{n=1} \approx 28 b^{2}$ compares well with the simulation result $\left\langle(\delta D)^{2}\right\rangle_{n=1} \approx 26 b^{2}$.

The higher states $n \geq 2$ display again lower fluctuations. This is in agreement with the interpretation that with growing kink density their repulsion and eventually mutual confinement become important.

Conclusion. - We have shown that the conceptually simple procedure of planar confinement transforms a simple mundane object - a helically coiled filamentinto a complex multistable, anomalously fluctuating filament. The statistical mechanics of this exotic object -the "squeelix" - can be qualitatively understood in terms of the motion of discrete particle-like entities corresponding to sharp curvature inversion points - called "twist kinks". At low twist kink concentrations they move almost freely along the chain and induce anomalously strong conformational fluctuations- notably deviating from wormlike chain behavior. The "squeelical" shapes formed under confinement range from almost ideally circular to wavy depending on the value of a single dimensionless "twist expulsion" parameter $\gamma=\frac{4 B \omega_{1}^{2}}{\pi^{2} C \omega_{3}^{2}}$. The latter parameter depends both on the chain's elastic moduli (flexural modulus $B$, twist modulus $C$ ) and the geometric properties (intrinsic curvature $\omega_{1}$ and twist $\left.\omega_{3}\right)$. For $\gamma>1$, the twist is curiously expelled from the chain. Under these conditions the squeelix becomes almost circular (up to minor end-effects) and the circularization probability can be dramatically enhanced. 
In the other limit $\gamma<1$ the squeelix comprises densely packed twist-kinks in its ground state which is now wavy.

We may speculate whether abnormal wavy shapes or enhanced closure of confined filaments found in literature are fingerprints of an otherwise hidden helical superstructures whose microscopic origin should be elucidated for each type of filament, case by case. Such peculiar behavior under confinement is observed for essential filaments like microtubules 7 , F-actin 28 and possibly for intermediate filaments 31. It has not escaped our attention that actin filaments circularize on stunningly small scales ( $~ 5 \mu m$ length rings) [28, 29] and exhibit wavy periodic tangent-correlation functions in narrow, flat -channels (cf. Fig .6 in [30) - both phenomena that neither can be understood within the naive WLC model. Similarly intermediate filaments under confinement show wiggly periodic shapes [31] suspiciously reminiscent of squeezed helices.

It is our feeling that the here illustrated phenomena of multistability, hyperflexibility and enhanced chain circularization are just the tip of an ice-berg and that floppy "squeelix" concept will help us to unravel a number of mysteries from biofilament realm hidden out in literature. We have evidence that additional lateral confinement of filaments in narrow channels, as encountered in microfluidic devices [31, further enhances the visibly wavy shapes for helical filaments and gives easier access to the underlying mechanical parameters. The potential of the 2-D and especially double confinement experiments (in microchannels) has been vastly underestimated so far. The "squeelix" phenomenology laid out here will serve us as a "dictionary" to decode these peculiar observations in forthcoming works.

Acknowledgment G.-M. Nam and N.-K. Lee acknowledge financial support of Korean Research Foundation via Grants NRF 2009-0084933 and NRF 2008-314-C00155. I.M.K. and A.J. acknowledge support from STAR exchange program and insightful discussions with Sarah Köster, Daniel Riveline and Albrecht Ott.

\section{REFERENCES}

[1] C. Lu, M. Reedy, and H.P. Erickson, J. Bacteriol. 182, $164(2000)$.

[2] F. van den Ent, L.A. Amos, and Jan Löwe, Nature, 413, 39 (2001).

[3] R. Kamiya and S. Asakura, J. Mol. Biol. 106, 167 (1976).

[4] E. Hasegawa, R. Kamiya, and S. Asakura, J. Mol. Biol. 160, 609 (1982).

[5] X. Li, J. Mol. Biol. 395, 327 (2010)

[6] H. Herrmann and U. Aebi, Annu. Rev. Biochem. 73, 749 (2004).

[7] P. Venier et al., J. Biol. Chem. 269, 13353 (1994).

[8] Mohrbach, H., A. Johner, and I. M. Kulić. Phys. Rev. Lett. 105, 268102 (2010); Eur. Biophys. J. 41, 217 (2012)

[9] C. W. Wolgemuth et al., Phys. Biol. 2, 189 (2005)

[10] Berg H. C. and Anderson R. A., Nature(London), 245, 380 (1973).
[11] A. Volodin, M. Ahlskog, E. Seynaeve, and C. Van Haesendonck, Phys. Rev. Lett. 84, 3342 (2000);

[12] S. M. Douglas, J. J. Chou, and W.M. Shih, Proc. Natl. Acad. Sci. 104, 6644(2007)

[13] L. Li et al , Angew. Chem. Int. Ed. 46, 5873 (2007)

[14] J. F. Currie, J. A. Krumhansl, A. R. Bishof and S. E. Trullinger, Phys. Rev. B. 22, 477 (1980).

[15] I. M. Kulić et al. Phys. Rev. E., 75, 011913 (2007).

[16] F. Wang and D. P. Landau, Phys. Rev. Lett. 86, 2050 (2001).

[17] D. Chrétien, F. Metoz, F. Verde, E. Karsenti, and R.H. Wade, J. Cell. Biol. 117, 1031 (1992).

[18] S. Asakura, G. Eguchi, and T. Iino, J. Mol. Biol. 16, 302 (1966).

[19] The solution is $\psi(s)=a m\left(\sqrt{a}\left(s-s_{0}\right) \mid \frac{\omega_{1}^{2}}{c a}\right)$ (elliptic amplitude function) where the constants of integration $a$ and $s_{0}$ are determined by the boundary conditions.

[20] Note that a complete twist expulsion at chain's ends is formally incompatible with the zero torque boundary condition, giving rise to small boundary layers close to filament's edges. For small deviations, Eq[5 implies $\vartheta^{\prime \prime}(\delta)-\vartheta(\delta) / \lambda^{2}=0$ (with $\delta=L / 2-s$ distance to the edge) supplemented by the boundary conditions $d \vartheta /\left.d \delta\right|_{\delta=0}=-2 \omega_{3}$ and $\lim _{\delta \rightarrow \infty} \vartheta=0$. Solving for the twist of the cHWLC gives $\psi(\delta)-\pi / 2=\frac{2}{\pi \sqrt{\gamma}} \exp (-\delta / \lambda)$. The width of the thin boundary layer is again given by the kink-size $\lambda$ - the only characteristic scale in the problem. The small deflection hypothesis is satisfied provided that $\gamma \gg 1$, i.e. deeply in the twist expulsion regime.

[21] G. Chirico and J. Langowski, Biopolymers 34, 415 (1994).

[22] In contrast the corresponding 3-D (free chain's) free energy appears rather tame and featureless, displaying no multiple local minima or barriers and lacking the complexity of its 2-D confined chain counterpart (data not shown).

[23] C.Lu, M.Reedy, and H.P. Erickson, J. Bacteriol. 182, 164 (2000).

[24] R. M. Macnab and M. K. Ornston, J. Mol. Biol. 112, 1 (1977).

[25] M. Caplow, R. L. Ruhlen, and J. Shanks, J. Cell Biol. 127, 779 (1994); M. I. Molodtsov et al., Proc. Natl. Acad. Sci. U.S.A. 102, 4353 (2005).

[26] B. Ghosh and A. Sain, Phys. Rev. Lett. 101, 178101 (2008).

[27] C. Zhou et al., Phys. Rev. Lett. 96, 120201 (2006).

[28] T. Sanchez, I. M. Kulić, and Z. Dogic, Phys. Rev. Lett. 104, 098103 (2010).

[29] J.X. Tang, J.A. Käs, J.V. Shah and P.A. Janmey, Eur. Biophys. J. 30, 477 (2001)

[30] S. Köster, D. Steinhauser and T. Pfohl, J. Phys. Condens. Matter 17, 4091, (2005)

[31] B. Nöding and S. Köster, Phys. Rev. Lett. 108, 088101 (2012). 